\title{
Florence Nightingale y Federico Rubio: reformadores de la Enfermería moderna española
}

\author{
Florence Nightingale and Federico Rubio: \\ nursing reformers modern spanish
}

\section{Florence Nightingale e Federico Rubio: reformadores enfermagem moderna española}

Elena Santainés Borredá ${ }^{1}$ Francisco Faus Gabandé2; Ramón Camaño Puig ${ }^{3}$ y Fernanda Sarturi ${ }^{4}$

${ }^{1}$ Enfermera Máster. Doctaranda en el programa de Enfermería de la Universidad de Valencia.

${ }^{2}$ Profesor Titular Doctor del Departament d'infermeria de la Universitat de València.

${ }^{3}$ Profesor Titular Doctor del Departament d'infermeria de la Universitat de València.

${ }^{4}$ Profa. Enfa. Ms. Universidade Federal de Santa Maria (UFSM). Palmeira das Missões/RS/Brasil

Santainés Borreda E; Faus Gabandé F; Camaño Puig R; Sarturi F. (2012) Florence Nightingale y Federico Rubio: reformadores de la Enfermería Moderna. Cultura de los Cuidados. (Edición digital) 16, 33. Disponible en: http://dx.doi.org/10.7184/ cuid.2012.33.06>

Correspondencia: Elena Santainés Borredá. Avd/Albaida no 4, piso 4º pta. 11ª Ontinyent 46870 (Valencia) ele0001.sb@gmail. $\mathrm{com} / \mathrm{Tel}: 650826684$

Recibido: 28/11/2011// Aceptado: 23/02/2012

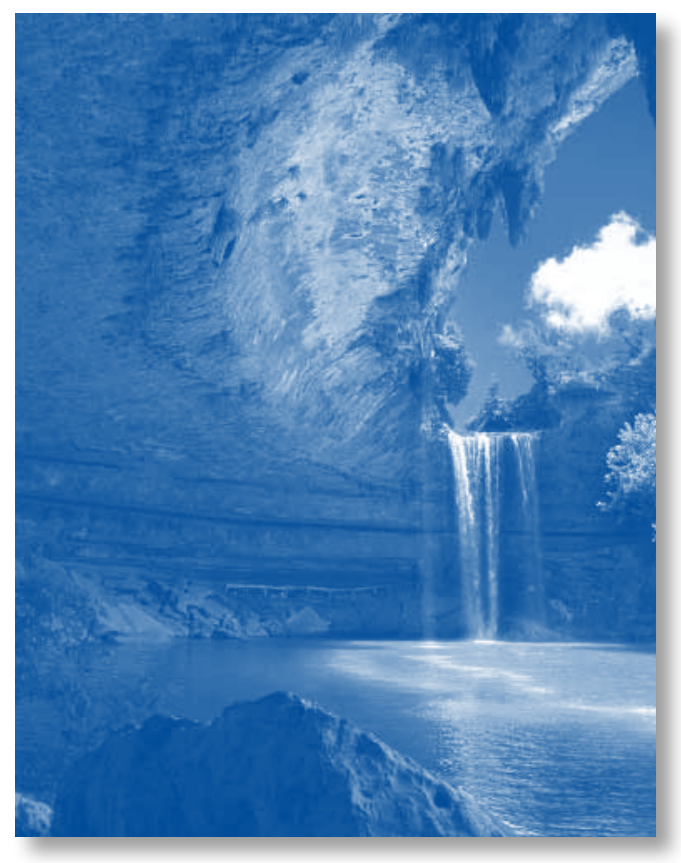

ABSTRACT

The surgeon Federico Rubio-Gali is mentioned in connexion with Florence Nightingale. In different publications giving him a prominence in the development of the modern
Spanish nursing profession. With this work we propose to analyze the influence that Nightingale could have had over Rubio-Gali. We want to analyse the life and works of the founder of the School of Nurses of Santa Isabel de Hungría establishing the intellectual temporary and spatial coincidences with the Lady of the Lamp. Our objective is to establish the parallelisms between both lives and examine the possible impact of Nightingale in the beginnings of the modern Spanish nursing. The analysis of primary and secondary documental sources was the technique chosen to compare the biography and works of Nightingale and RubioGali. Some of the similarities founded, e.g. in the syllabuses of their respective schools and different health measures would confirm the influence of Nightingale in Rubio-Gali, nevertheless, only in Hernández (1996), Nightingale is recognised as a key point in the contributions of Rubio-Gali.

Keywords: History, Nursing, Nightingale, Rubio-Gali. 


\section{RESUMO}

Em diferentes bases de dados aparecem publicações que mencionam o cirurgião Federico Rubio em relação a enfermeira Florence Nightingale, dando-lhe um protagonismo na enfermagem española. Mediante este trabalho nos propomos a analizar a influência que Nightingale pode ter tido sobre Rubio. Partindo da vida e obra do fundador da Escola de Enfermagem de Santa Isabel da Hungría, valorizaremos as coincidencias tempo-espaciais con a Dona da Lâmpada. Nossos objetivos visam establecer paralelismos entre ambas vidas, assim como repercutir a percepção de Nightingale na enfermagem española moderna. A análise documental foi a técnica escolhida para cadastrar a bibliografía e obra de Nightingale e Rubio, recorrendo tanto a fontes de informação primárias como secundárias. Alguns dos dados obtidos tais como a similaridade nos papéis de estudos e de suas respectivas escolas e as medidas sanitárias relacionadas a Saúde Pública, poderiam reinterar a influência de Nightingale sobre Rubio. Estes exemplos, entre outros, mostram coincidencias y similaridades entre a enfermagem difundida por Nightingale e o plano proposto por Rubio. No entanto, somente Hernandez (1996), considerada Nightingale um ponto chave nas contribuições de Rubio.

Palavras-chave: História, Enfermagem, Nightingale, Rubio.

\section{RESUMEN}

En diferentes bases de datos aparecen publicaciones donde se menciona al cirujano Federico Rubio en relación con la enfermera Florence Nightingale, otorgándole un protagonismo en la enfermería moderna española. Mediante este trabajo proponemos analizar la influencia que Nightingale pudo haber tenido en Rubio. Partiendo de la vida y obra del fundador de la Escuela de Enfermeras de Santa Isabel de Hungría, valoraremos las coincidencias temporo-espaciales con la Dama de la Lámpara. Nuestros objetivos persiguen establecer paralelismos entre ambas vidas, así como reflejar la repercusión de Nightingale en la enfermería española moderna. El análisis documental fue la técnica escogida para contrastar la biografía y obra de Nightingale y Rubio, recurriendo tanto a fuentes de información primarias como secundarias. Algunos de los datos obtenidos tales como la similitud en los planes de estudios de sus respectivas escuelas y las medidas sanitarias relacionadas con la Salud Pública, podrían ratificar la influencia directa de Nightingale en Rubio. Estos ejemplos, entre otros, muestran coincidencias y similitudes entre la enfermería difundida por Nightingale y el plan propuesto por Rubio. Sin embargo, sólo en Hernández (1996), Nightingale es considerada un punto clave en las aportaciones de Rubio.

Palabras clave: Historia, Enfermería, Nightingale, Rubio.

\section{INTRODUCCIÓN}

El presente trabajo pretende ofrecer una reflexión sobre nuestra historia, nuestras raíces y nuestros valores actuales, centrándonos en una época determinada, los inicios de la Enfermería Moderna en España. En opinión de Campos (2005) historiadores como Gutiérrez, Álvarez Sierra, Sánchez Cuesta e incluso el mismo Laín Entralgo (1974) representan ejemplos significativos de relatos biográficos de Rubio Galí en los que se destaca su éxito dentro del campo de la medicina, destacando en sus escritos una exaltación como precursor de la Cirugía Española y describen un Rubio que fundó la primera escuela de enfermeras de 
la España moderna, la Escuela de Sta. Isabel de Hungría en 1896.

Ante esta institución personalizada por el doctor Rubio, las enfermeras nos preguntamos ¿de dónde emanan realmente las aportaciones de su propuesta? Esta cuestión puede plantear otras como: ¿quién fue la verdadera fundadora de la enfermería moderna en España? ¿Se ha investigado sobre el origen ideológico de las aportaciones de Rubio en el campo de la enfermería? O en su caso, ¿pudo influir la Florence Nightingale en la iniciativa de Rubio? A la hora de responder a estos interrogantes no hay que dejar de lado la consideración de Donahue (1985) sobre la existencia de cronistas como Cook, Strachey, Goldsmith y Woodham-Smith que descontextualizan a la enfermera Nightingale de su marco histórico. Es por ello, que se recurrió al estudio y análisis de archivos que relatan los acontecimientos desde perspectivas muy diferentes; encontrando, en algunos casos, biografías centradas en una faceta concreta de la vida de los personajes, minimizando o restándole importancia a otros aspectos que pudiesen tener relevancia para nuestra historia. Se partió de una valoración acerca de las coincidencias temporo-espaciales entre $\mathrm{Ni}$ ghtingale y Rubio y la posible influencia de la Dama de la Lámpara en las aportaciones del Cirujano respecto a los cuidados y, concretamente, en la formación de las enfermeras modernas en España.

Partiendo de esta idea, se plantean dos objetivos: en primer lugar y una vez estudiada la biografía de F. Nightingale y de F. Rubio se analizan los posibles paralelismos de ambas vidas y la posible repercusión que Nightingale pudo ejercer sobre los pilares de la enfermería española moderna, sirviéndonos de descripciones de diversos autores, citas y aportaciones tanto de Rubio como de Nightingale que muestran una influencia de nuestra referente profesional de los cuidados sobre la que fue la primera escuela de enfermeras modernas en España.

\section{METODOLOGÍA}

La investigación documental ha sido la técnica escogida para el análisis y comparación de la vida y obra de Nightingale (1820-1910) y Rubio (1827-1902); consistiendo en revisar la biografía de ambos dentro de su contexto sociopolítico y profesional. De esta forma se puede apreciar, desde una perspectiva más objetiva, su acción y repercusión a nivel social y sanitario. Se trató de reconstruir una semblanza en la que se conectaba la obra y la época en la que vivió cada individuo, considerándolo como un todo, de la misma forma en la que se parte de la idea holística del ser humano en la elaboración de un plan de cuidados enfermeros. Cada una de sus vidas se tomó como hilo conductor para intentar explicar los inicios de la Enfermería Moderna en España, procurando conectar y mostrar interacciones entre ambos profesionales.

Para ello, nos servimos tanto de descripciones detalladas de diversos autores como de aportaciones de ambos protagonistas. Relatos como los de Palma y Palma (2002), Campos (2005), Marset y Sáez (2003) y un largo etcétera sobre F. Rubio y otros centrados en la vida de F. Nightingale como el de Attewell (1998), Morillo (2005), Hanzeliková, Carabaño, Torresano, et al. (2005), Selanders (2010), entre otros, nos sirvieron para crear una cronología que trataba de armonizar los hechos histórico-temporales y los trabajos de ambos profesionales. Para poder evidenciar las repercusiones cultivadas por la enfermera sobre el cirujano tomamos como hilo conductor su libro Notas sobre Enfermería. Qué es y qué no es (2004), así como otras fuentes que describen lo acaecido en la Guerra de Crimea; como Florence 
Nightingale and The Crimea (1854-1855) de Coates (2000).

\section{RESULTADOS}

Con la información recogida se procedió a reflejar la cronología gráfica de ambos protagonistas, evidenciando las coincidencias tanto en espacio como en tiempo. Uno de los hallazgos que pudiese resultar interesante, es por ejemplo la creación de la Escuela de Enfermeras en el Hospital de St. Thomas en Inglaterra en 1860 , momento en el que F. Rubio se encuentra exiliado en Londres (Campos, 2004). Además, de los diversos textos que recogen la historia de la enfermería española se desprende que Florence Nightingale no parece estar considerada como un pilar base para Federico Rubio en la creación de la Escuela de Enfermeras de Santa Isabel de Hungría, no relacionándose la vida de ambos, sino que se consideran dos personajes independientes, referenciando que "sobre la Escuela de Santa Isabel de Hungría (1894), nos resulta dificultosa su investigación" (Eseverri Chaverri, 1984, pp. 296) y afirmando que las ideas de Nightingale "sus teorías, en España, apenas si influyeron en este periodo que nos ocupa, es decir, 1850-1915" (Eseverri Chaverri, 1984, pp. 298). Teniendo en cuenta que dicho historiador no nombra a Rubio, es posible

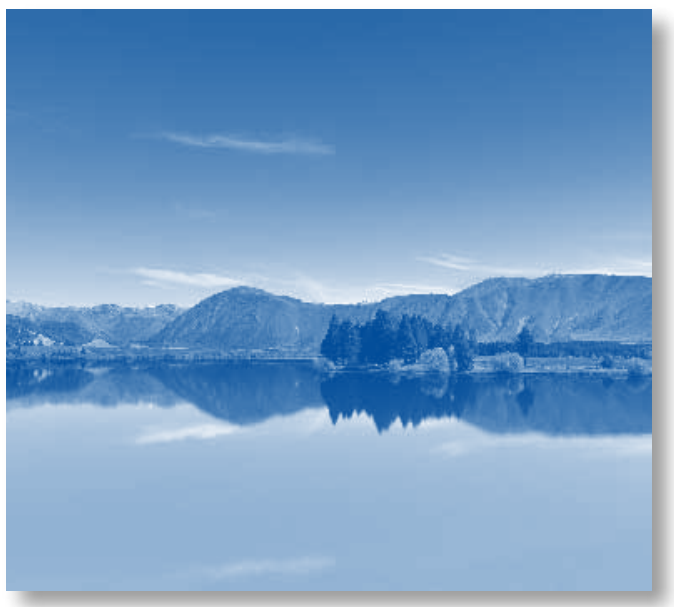

plantearnos la idea de que no ha considerado relevante el periodo de exilio en Londres del cirujano en 1860.

Otros autores como Donahue (1985) muestran de forma gráfica en su obra las ciudades que entre 1860 y 1910 tomaron como referencia el sistema de Nightingale para la formación de enfermeras, algunas de ellas son: Sta. Catharines en 1864, New Haven en 1873, Dublín en 1879, Berlín en 1886, Roma en 1894, Beirut en 1906 y un largo etcétera, y nos parece llamativo que España no aparezca citada con la Escuela de Rubio creada en 1896. En este sentido el trabajo de Domínguez Alcón (1986), parece no considerar a Nightingale como fuente de inspiración de la Escuela de Enfermeras de Rubio en España.

Otras publicaciones más actuales indican que Rubio da gran importancia al médico higienista en la construcción de un ferrocarril considerando necesario un barracón en cada colonia con camas, colchones y demás aparataje hospitalario, es decir, la modernización de la sanidad hacia una buena Salud Pública (Herrera, 2002). Esta aportación es bastante similar a las medidas establecidas por Nightingale años atrás: "una mala sanidad, una mala arquitectura y una mala administración a menudo hacen imposible cuidar" (Nightingale, 2004, pp.2), afirmación que manifiesta la importancia de una adecuada implementación enfermera en Salud Pública; además de conocer que Nightingale fue la primera que estableció una conexión directa entre higiene y curación (Mulrain, 2010). Siguiendo con la misma idea, Rubio publica cinco reseñas anuales entre los años 1881-1885 en donde detalla las medidas en relación a la lavandería, ropa y ventilación entre otras, acompañadas de una descripción detallada del funcionamiento y de las intervenciones llevadas a cabo en el Instituto de Terapéutica Operatoria del Hospital de la Princesa, 
fundado por el mismo en Madrid en 1880. Nightingale se puede ver reflejada en los escritos de F. Rubio cuando este expresa la necesidad de sanear la atmósfera del entorno del enfermo. Báguena (2003, pp. 123) cita unas palabras textuales del cirujano en las cuales se refiere que "el vapor de agua que hemos examinado bajo una forma líquida encierra sustancias nocivas para la respiración de los enfermos y presupone una atmósfera de todo punto antihigiénica y que conviene sanear, poniendo en práctica y vigor las leyes higiénicas en mal hora quebrantadas". La enfermera apuntaba en sus escritos previos a Rubio, que "la primera regla de la enfermería es mantener el aire que respira el paciente, tan puro como el aire de fuera sin que el paciente se resfríe [...] El aire estancado es el más propicio para cultivar la viruela, escarlatina, difteria o lo que uno quiera" (Nightingale, 2004, pp. 6-7).

Por último, entre los hallazgos encontrados relacionados con los planes de estudios consideramos de importancia resaltar que Nightingale fue una de las promulgadoras de los planes de estudio para la primera Escuela de Medicina Militar del Reino Unido en Fort Pitt fundada en 1860 (Attewell, 1998). Tampoco se puede dejar de lado los resultados obtenidos que informan de los aspectos de carácter administrativo de la Escuela de Enfermeras del Hospital St. Thomas en Londres y la Escuela de Enfermeras de Santa Isabel de Hungría en España fundadas por Nightingale y Rubio, respectivamente, descritos en los trabajos de Santamaría (2007) y Quintairos (2008). Todas estas citas revelan cierta conexión de la obra de la enfermera Nightingale con la del cirujano Rubio, aunque presumimos que hay más datos que es necesario investigar y que pueden ir estableciendo la relación que pudiese existir entre sus obras y que, por supuesto, merecen un análisis más exhaustivo.

\section{DISCUSIÓN}

La información recogida y analizada hasta el momento nos indica que la asociación entre el exilio de Rubio y el momento en el que Nightingale crea la primera escuela de enfermeras en Londres, es el dato más enunciado por historiadores y otros profesionales, siendo en la mayoría de los casos la única alusión que se hace a F. Nightingale en relación a la enfermería moderna en España, lo que aparentemente parece ser nada más que una coincidencia para sus narradores. No obstante, hay aspectos que pueden ser analizados y discutidos, como es el caso de las medidas higiénico-sanitarias instauradas por el cirujano en el Instituto de Terapéutica Operatoria. Medidas, todas ellas, que parecen ser análogas a las establecidas por Nightingale como enfermera jefe en el Hospital de Scutari, durante la guerra de Crimea. Los resultados, como es el caso del saneamiento de la atmósfera del enfermo descrito por $\mathrm{Ru}$ bio nos hacen pensar en que nuestra hipótesis puede ser ratificada, es decir, que parte de las aportaciones de Rubio en el campo de la Enfermería Moderna no fuesen tan novedosas, pues coinciden con los conocimientos elaborados por Nightingale años atrás

Al mismo tiempo es necesario tomar en consideración la relevancia a las aportaciones de Nightingale en el ámbito de la cirugía, recordemos que estuvo como enfermera jefe en el hospital de Scutari durante la Guerra la Crimea y gran parte de sus pacientes pudieron requerir no sólo cuidados higiénicos y de alimentación, sino también tratamiento de heridas de guerra, de infecciones contagiosas graves y, en los peores casos, intervenciones quirúrgicas. El hecho de que fuera una de las involucradas en los planes de estudio de la primera Escuela de Medicina Militar del Reino Unido puede ser prueba de su valía $y$, dado que hace referencia al campo de acción de F. Rubio, pensamos que pudo 
ser un hecho que interesase en gran medida al cirujano español. Otro de los aspectos para deliberar que puede evidenciar la relación entre Nightingale y Rubio hace mención a la configuración administrativa de sus respectivas escuelas. En los trabajos de Santamaría (2007) y Quintairos (2008) se puede comprobar la similitud en cuanto a los años de formación, edad de las estudiantes, educación recibida y materias troncales, además de la alusión por parte de ambos profesionales a la educación tanto teórica como práctica.

Sin embargo, la discusión resulta más difícil si profundizamos en las publicaciones más actuales indexadas en diferentes bases de datos, pues aparece siempre la relación con $\mathrm{Ni}$ ghtingale como algo secundario, lo que conlleva el otorgarle a Rubio un protagonismo que pudiese ser equívoco en cuanto a los inicios de la Enfermería Moderna en España (Herrera, 2002; Vázquez, 2005; Poyato, García y Álvarez, 2007; Herrera, 2009). Autores como Hernández, Delgado, Pinar, et al., (1994); Hernández, Pinar y Moreno (1996-1997) y Hernández, Gallego, Alcaraz, et al. (1997) reseñan únicamente que Rubio, siendo testigo del acontecimiento de la creación de la Escuela de Nightingale mientras estaba en Inglaterra -1860 a 1870- fue quién fundó en 1896 la Primera Escuela de Enfermería en España. Sin clarificar de forma evidente hasta que punto llega esa atribución o si, por el contrario, se trata de una contribución directa de Nightingale en España a través de un profesional de otra disciplina que tuvo la oportunidad de transmitirlo a nuestra sociedad.

Para concluir, con los hallazgos descritos es posible pensar en la existencia de sesgos en la historia de la Enfermería Moderna en España. No hay que olvidar que la historia es de quién la escribe, siendo la deliberación y la dimensión crítica de diferentes fuentes, imprescin- dibles para poder lograr una mayor objetividad en los hechos que fundamentan nuestro pasado $\mathrm{y}$, como consecuencia, nuestra calidad como profesionales. Llegando a conseguir las bases evidentes de los cuidados desde la perspectiva histórica propia de la enfermería y no de otras disciplinas, como en este caso la medicina de Rubio y Galí. Por ello, planteamos un estudio más minucioso que permita verificar esta breve aportación que pretende despertar interés en aquellos profesionales que quieran dar un valor significativo a sus raíces y a su profesión enfermera.

\section{REFERENCIAS BIBLIOGRÁFICAS}

- Attewell, A. (1998). Florence Nightingale. Perspectivas, revista trimestral de educación comparada, XXVIII (1), 173-189.

- Báguena, M.J. (2003). La Microbiología en la obra de Federico Rubio. En Carrillo Martos, J.L., Medicina y Sociedad en la España de la Segunda Mitad del Siglo XIX (pp. 119-128). Puerto de Santa María: Ayuntamiento del Puerto de Santa María y Asociación para la Formación. Investigación y Asistencia Médica de Andalucía "Federico Rubio".

- Campos, R. (2004). Curar y gobernar. Medicina y liberalismo en la España del siglo XIX. Monlau, Rubio y Giné. Tres Cantos (Madrid): Nivola Libros y Ediciones, S.L.

- Campos, R. (2005). Algunas reflexiones sobre la biografía divulgativa. Los casos de Monlau, Rubio y Giné. Asclepio, 57 (1), 149-166.

- Coates, T (2000). Florence Nightingale and The Crimea, 1854-1855. Guildford (Surrey): The Stationery Office.

- Domínguez Alcón, C. (1986). Los cuidados y la profesión enfermera en España. Madrid: Pirámide.

- Donahue, M. P. (1985). Historia de la Enfermería. España: Ediciones Doyma.

- Eseverri Chaverri, C. (1984). Historia de la enfermería española e hispanoamericana. Barcelona: Salud, S.A.

- Hanzelikovà, A; Carabaño, M.J; Torresano, B. y García, M.R. (2005). Florence Nightingale y el contexto históri- 
co-sociocultural de su época. Cultura de los Cuidados, año IX (18), 24-33.

- Hernández, F; Delgado, A; Pinar, M.E. y Villahoz, M.C. (1994). Orígenes “modernos" de la enfermería española: primera escuela de enfermería en España, 1896. JANO, XLVII (1098), 55-57.

- Hernández, F; Gallego, R; Alcaraz, S; González, J.M. (1997). La enfermería en la Historia. Un análisis desde la perspectiva profesional. Cultura de los Cuidados, (2), 21-35.

- Hernández, F; Pinar, M.E. y Moreno, M.A. (1996-1997). Memoria de un centenario. Primera Escuela de Enfermería Santa Isabel de Hungría. Híades, Revista de Historia de la Enfermería, (3-4), 189-195.

- Herrera, F. (2002). Un acercamiento a la obra de Federico Rubio y Galí (1827-1912). Revista de Historia de El Puerto, (29), 63-88.

- Herrera, F. (2009). La enfermería en la obra de Concepción Arenal y de Federico Rubio. Enfermería Gaditana, 4 (2), 37-39.

- Marset, P. y Sáez, J.M. (2003). Medicina, estado y sociedad en la España de la segunda mitad del siglo XIX. En Carrillo Martos, J.L., Medicina y Sociedad en la España de la Segunda Mitad del Siglo XIX (pp. 1-34). Puerto de Santa María: Ayuntamiento del Puerto de Santa María y Asociación para la Formación. Investigación y Asistencia Médica de Andalucía "Federico Rubio".

- Mulrain, J. (2010). Porgrama de la Exposición de Florence Nightingale ExhibitTM. La enfermera 39a . En honor del $100^{\circ}$ aniversario de su muerte en 2010. Panamá: Museo Nacional de Antropología.

- Lain Entralgo, P. (1974). Historia universal de la Medicina. Barcelona: Salvat Editores, S.A.

- Morillo, R.M. (2005). Florence Nightingale en la Guerra de Crimea. Un legado para la enfermería del siglo XXI. Temperamentvm, 2. Disponible en http://www.index-f. com/temperamentum/tn2/t0128.ph

- Nightingale, F (2004). Notas sobre Enfermería. Qué es y qué no es. Barcelona: Masson, S.A.

- Palma, F. y Palma, C. (2002). Historia de las reseñas de los ejercicios del Instituto de Terapéutica Operatoria del Hospital de la Princesa (1881-1885). Salamanca: Europa artes gráficas, S.A.

- Poyato, J.M; García, M.M. y Álvarez, M.F. (2007). Federico Rubio y Galí, "Príncipe de la Cirugía”, y la urología en la Sevilla del Siglo XIX. Archivos Españoles de Urología, $60(8), 931-942$.

- Quintairos, S. (2008). Del practicante a la enfermera. 150 años de desarrollo profesional: el papel de los colegios profesionales. Universidad de A Coruña. Departamento de Ciencias da Saúde. España.

- Riera, J. (1973). La introducción en España del método antiséptico de Lister. Valladolid: Universidad de Valladolid, Ediciones del Seminario de Historia de la Medicina.

- Santamaría, M.B. (2007). Evolución histórica de la enfermería en Cardiología. Enfermería en Cardiología, año XIV (40), 21-28.

- Selanders, L. (Eds.). (2010). Florence Nightingale by name Lady with the Lamp. En Encyclopaedia Britannica [versión electrónica]. New York: Enclicopaedia Britannica Inc, http://www.britannica.com/ EBchecked/ topic/415020/Florence-Nightingale\#

- Vázquez, F. (2005). Instituto de Terapéutica Operatoria (1880-1939). Instituto Rubio y Galí, Instituto Moncloa. Contribución a las especialidades médicas y enfermería en España. Anales de la Real Academia Española, tomo CXXII, 411-430. 